\title{
Molecular characteristics of the inhibition of human neutrophil elastase by nonsteroidal antiinflammatory drugs
}

\author{
Kooil Kang ${ }^{1,4}$, Sung Jun Bae', Woo Mi Kim, \\ Dae-Heui Lee ${ }^{1}$, Unsuck Cho', Myung Huck Lee', \\ Mu-Sang Lee ${ }^{2}$, Sang-il Nam², Klaus E. Kuettner ${ }^{3}$ \\ and David E. Schwartz ${ }^{3}$ \\ ${ }^{1}$ Department of Pharmacology, Kosin University Medical College, \\ Pusan, Korea \\ ${ }^{2}$ Department of Chemical Education, Kyungpook National Univer- \\ sity, Taegu, Korea \\ ${ }^{3}$ Department of Biochemistry, Rush-Presbyterian-St. Luke's \\ Medical Center, Chicago, IL., U.S.A. \\ ${ }^{4}$ Corresponding author: Tel, +82-51-240-6420; \\ Fax, +82-51-240-6488, +82-51-241-5458; \\ E-mail,kooil1@ hotmail.com, kooil1@chollian.net
}

Accepted 19 September 2000

Abbreviations: NSAIDs, nonsteroidal antiinflammatory drugs; HNElastase, human neutrophil elastase; DTT, dithiothreitol; DMSO, dimethylsulfoxide

\begin{abstract}
Nonsteroidal antiinflammatory drugs(NSAIDs) are known as clinically effective agents for treatment of inflammatory diseases. Inhibition of cyclooxygenase has been thought to be a major facet of the pharmacological mechanism of NSAIDs. However, it is difficult to ascribe the antiinflammatory effects of NSAIDs solely to the inhibition of prostaglandin synthesis. Human neutrophil elastase (HNElastase; HNE, EC 3.4.21.37) has been known as a causative factor in inflammatory diseases. To investigate the specific relationship between HNElastase inhibition and specificity of molecular structure of several NSAIDs, HNElastase was purified by Ultrogel AcA54 gel filtration, CM-Sephadex ion exchange, and HPLC (with TSK 250 column) chromatography. HNElastase was inhibited by aspirin and salicylate in a competative manner and by naproxen, ketoprofen, phenylbutazone, and oxyphenbutazone in a partial competative manner, but not by ibuprofen and tolmetin. HNElastase-phenylbutazone-complex showed strong Raman shifts at 200, 440,1124, 1194, 1384, 1506, and $1768 \mathrm{~cm}^{-1}$. The Raman bands 1194, 1384, and 1768 $\mathrm{cm}^{-1}$ may represent evidences of the conformational change at $-\mathrm{N}=\mathrm{N}-\Phi$ radical, pyrazol ring, and $-\mathrm{C}=\mathrm{O}$ radical of the elastase-drug complex, respectively.
\end{abstract}

Phenylbutazone might be bound to HNElastase by ionic and hydrophobic interaction, and masked the active site. Inhibition of HNElastase could be another mechanism of action of NSAIDs besides cyclooxygenase inhibition in the treatment of inflammatory diseases. Different inhibition characteristics of HNElastase by NSAIDs such as aspirin, phenylbutazonelike drugs and ineffective drugs could be important points for drawing the criteria for appropriate drugs in clinical application.

Keywords: NSAIDs, human neutrophil elastase, inflammatory diseases, Raman shifts.

\section{Introduction}

Human neutrophil elastase (HNElastase, EC 3.4.21.37), is a causative factor in inflammatory diseases such as emphysema and rheumatoid arthritis (Janoff, 1972; Oholson and Olsson, 1977; Starkey, 1980; Fritz et al., 1986; Abramsom \& Weissman, 1989), HNElastases are regulated by plasma proteinase inhibitors such as alpharproteinase inhibitor and alpha ${ }_{2}$-macroglobulin (Janoff, 1972; Oholson and Olsson, 1977; Starkey, 1980; Fritz et al., 1986). Nonsteroidal antiinflammatory drugs (NSAIDs) are known as clinically effective agents for treatment of inflammatory diseases such as rheumatoid arthritis (Lentin et al., 1987; Vane et al., 1987 and 1995; Insel, 1996). The mechanism of action has been known that NSAIDs inhibited cyclooxygenase (Vane et al., 1987 and 1995; Insel, 1996). However, it is difficult to ascribe the antirheumatoid effects of aspirin-like drugs solely to the inhibition of prostaglandin synthesis (Insel, 1996) since HNElastase shows different sensitivity to the NSAIDs (Kang et al., 1996). Specific interaction between the enzyme and the individual drug may be present. Total amino acid sequence of HNElastase was reported by Sinha (Sinha et al., 1987) and Bode (Bode et al., 1986). Navia (Navia et al., 1989) reported the X-ray crystallographic structure. In comparison with other methods including IR or NMR, it is reasonable to investigate the mechanism of drug action by conformational change of the elastase-drug complex with Raman spectroscopic method. Since this method has the special advantage with only a small interference from the main biological solvent, water (Keller et al., 1994). In this paper, we describe the characteristics of HNElastase inhibition by NSAIDs. We also propose a possible molecular, pharmacological inhibition mechanism of HNElastase by 
phenylbutazone according to the analysis of Raman spectroscopic data and visualized molecular model of human neutrophil elastase.

\section{Materials and Methods}

\section{Materials}

$\mathrm{N}$-Succinyl-Ala-Ala-Ala-p-nitroanilide (SANA), N-succinylAla-Ala-Pro-Phe-p-nitroanilide (SAPNA), oxyphenbutazone, phenylbutazone, ibuprofen, salicylate, aspirin, ketoprofen, ibuprofen, tolmetin, indomethacin, sulindac, and Hypaque-Ficoll were Sigma products. Tris, glycine, and sodium dodecyl sulfate were Bio-Rad products. Ultrogel AcA54 and CM-Sephadex C-25 were Pharmacia products. Spectro pore 2 dialysis membrane was a product of Spectrum Medical Industries INC., L.A., U.S.A.. PM 10 ultrafiltration membrane was the product of Amicon Co.. All other chemicals were of the highest quality obtainable.

\section{Purification of human neutrophil elastases}

Human neutrophils were isolated from whole blood (acquired from the blood bank of Kosin Medical Center) by Ficoll-hypaque step gradient centrifugation at $200 \times \mathrm{g}$ for $20 \mathrm{~min}$ and washed three times with $50 \mathrm{mM}$ Tris-Cl buffer, pH 7.3 (Voyum, 1968; Jeung et al., 1987).

The method of purification of HNElastase was a modified method by Baugh and Travis (1976). In brief, isolated neutrophils were suspended in $0.5 \mathrm{M}$ Tris- $\mathrm{Cl}$ buffer, $\mathrm{pH} 7.3$, containing $1.5 \mathrm{M} \mathrm{NaCl}$ and $50 \mathrm{mM} \mathrm{CaCl}_{2}$, then homogenized with Polytron for $30 \mathrm{sec}$ at $4^{\circ} \mathrm{C}$ and centrifuged at $30,000 \mathrm{~g}$ for $60 \mathrm{~min}$. The supernatant was then collected and kept at $4^{\circ} \mathrm{C}$. The extract was chromatographed through an AcA54 gel filtration column equilibrated with $50 \mathrm{mM}$ of Tris-Cl buffer containing 150 $\mathrm{mM}$ of $\mathrm{NaCl}$. Obtaining enzyme profile, HNElastase-rich fractions were pooled, concentrated, and dialyzed against $50 \mathrm{mM}$ of $\mathrm{NaAc}, \mathrm{pH} 4.5$, containing $150 \mathrm{mM} \mathrm{NaCl}$.

Elastase-rich fraction prepared by Ultrogel AcA54 gel filtration was chromatographed again with a CM-Sephadex ion exchange column equilibrated with $50 \mathrm{mM} \mathrm{NaAc}$ buffer, $\mathrm{pH} 4.5$, containing $150 \mathrm{mM}$ of $\mathrm{NaCl}$. Bound protein was eluted by a linear salt gradient $(0.15-1 \mathrm{M})$ in the same buffer.

Elastase-rich fractions were pooled, concentrated, and dialyzed against $50 \mathrm{mM}$ Tris- $\mathrm{Cl}, \mathrm{pH}$ 4.5, containing 150 $\mathrm{mM}$ of $\mathrm{NaCl}$. HNElastase purified by ion exchange chromatography was chromatographed again with $\mathrm{CM}$ Sephadex ion exchange column equilibrated with 50 $\mathrm{mM} \mathrm{NaAc}$ buffer, $\mathrm{pH} 4.5$, containing $150 \mathrm{mM}$ of $\mathrm{NaCl}$. Bound protein was eluted by a linear salt gradient (0.3$0.8 \mathrm{M}$ ) in the same buffer. Four peaks of HNElastase were separated by this shallow gradient. Four peaks of HNElastase-rich fractions were collected separately, and each peak was pooled, concentrated, and dialyzed against $50 \mathrm{mM}$ Tris- $\mathrm{Cl}$ containing $150 \mathrm{mM}$ of $\mathrm{NaCl}, \mathrm{pH} 7.3$, labeled each peak as E-1, E-2, E-3, and E-4, then kept at $-20^{\circ} \mathrm{C}$ until use.

The sample for Raman experiment was purified again from P-4 (least molecular weighted E4 elastase) by HPLC with TSK $250 \mathrm{gel}$ filtration column equilibrated with 50 $\mathrm{mM}$ Tris- $\mathrm{Cl}$ containing $0.8 \mathrm{M}$ of $\mathrm{NaCl}, \mathrm{pH}$ 7.3. Finally purified HNElastase was analyzed by SDS-PAGE.

\section{Elastase assay}

Elastase assay was carried out in $200 \mu \mathrm{l}$ of reaction medium (in 96-well microplate) containing 60-150 mM $\mathrm{NaCl}, 50 \mathrm{mM} \mathrm{CaCl}, \mathrm{pH} 7.3$ with or without inhibitor depending upon the design of the experiment (Kang, 1985). After 10-60 $\mu \mathrm{l}$ of elastase and substrate (SANA, 1-4 mM depend upon the design of experiment), was added to the reaction medium, then it was incubated at $37^{\circ} \mathrm{C}$ for an appropriate period (usually 10-40 min depending on the nature of experiment). Elastase activity was measured spectrophotometrically at $410 \mathrm{~nm}$ by monitoring the concentration of liberated p-nitroaniline, using a Titertek Multiskan Spectrophotometer (MCC/ 340, Flow laboratories, Switzerland).

\section{Preparation of drug bound elastase}

Elastase-phenylbutazone complex was prepared by the previously described method (Kang, 1998). Briefly, 500 $\mu \mathrm{g}$ of purified elastase was placed in $200 \mu \mathrm{g}$ of $50 \mathrm{mM}$ Tris buffer, $\mathrm{pH} 7.3$, containing $5 \mathrm{mM} \mathrm{CaCl}, 150 \mathrm{mM}$ of $\mathrm{NaCl}$, and $20 \%$ of dimethylsulfoxide (DMSO). After $24 \mu \mathrm{g}$ of $0.5 \mathrm{mM}$ phenylbutazone was added to the reaction medium containing HNElastase, then it was incubated at room temperature for $30 \mathrm{~min}$. The mixture of drug and elastase sample was placed in Spectropore 2 membrane, then dialyzed three times against one liter of 50 $\mathrm{mM}$ Tris- $\mathrm{Cl}$ buffer, $\mathrm{pH} 7.3$ at $4^{\circ} \mathrm{C}$. The dialyzed sample was frozen and kept at $-20^{\circ} \mathrm{C}$ until use.

\section{Raman experiments}

Raman spectra were recorded in melting point capillaries using Laser Raman Spectrophotometer System (Sepex $1403,0.85 \mathrm{~m}$ double spectrometer equipped by RCA-C 31034 phototube and DM 3000 software) (Kang, 1998). All spectra were recorded using the $514.5-\mathrm{nm}$ laser wavelengths for excitation. Data were collected at 1 $\mathrm{cm}^{-1}$ intervals with $0.3 \mathrm{~s}^{-1}$ integration time and $4 \mathrm{~cm}^{-1}$ spectral slit width.

\section{Molecular modeling from crystallographic datum and sequence information}

Two available crystallographic data files (Bode et al., 1986; Navia et al., 1989) of human neutrophil elastase were available from the Protein Data Bank (PDB) in Brookhaven National Laboratory, New York. The PDB 
covers atomic coordinates, structure factors and phases from different studies of macromolecules. In this description, we applied the Navia's PDB datum instead of Bode's since the Navia's PDB crystallographic datum was acquired from the elastase crystal crystallized after the activity was inhibited by a smaller molecular weight of inhibitor.

The molecular model was constructed with a molecular visualization program (Roger Sayler Biomolecular Structure, Glaxo Research and Development, Greenford, Middlesex, UK) using Navia's PDB datum of crystallographic information of human neutrophil elastase. The details of specification of drawing each model was described in our previous paper (Kang, 1998).

\section{Results}

\section{Purification of four different HNElastase isozymes}

Four elastase isozymes were separated effectively (usually not separated well) by three steps of purification procedure including Ultrogel AcA 54 gel filtration and two CM-Sephadex ion exchange chromatography (linear salt gradient 0.15-1 $\mathrm{M}$ and 0.3-0.8 $\mathrm{M} \mathrm{NaCl}$ ). Figure 1 showed the chromatography of CM-Sephadex ion exchange column eluted with shallow salt gradient. For the Raman experiments, the elastase isozyme (E-4) was chromatographed again by HPLC with TSK-250 gel filtration column and the peak4 was confirmed as a HNElastase isozyme, E-4 (Figure 1B). This HNElastase E-4 showed single band in SDS-PAGE (Figure 1B).

\section{Inhibition of HNElastase by NSAIDs}

Four different HNElastase isozymes (E-1, E-2, E-3, E4), separated by CM-Sephadex ion exchange chromatography, were tested for inhibition of elastase activity. Figure 2 showed that four isozymes revealed different
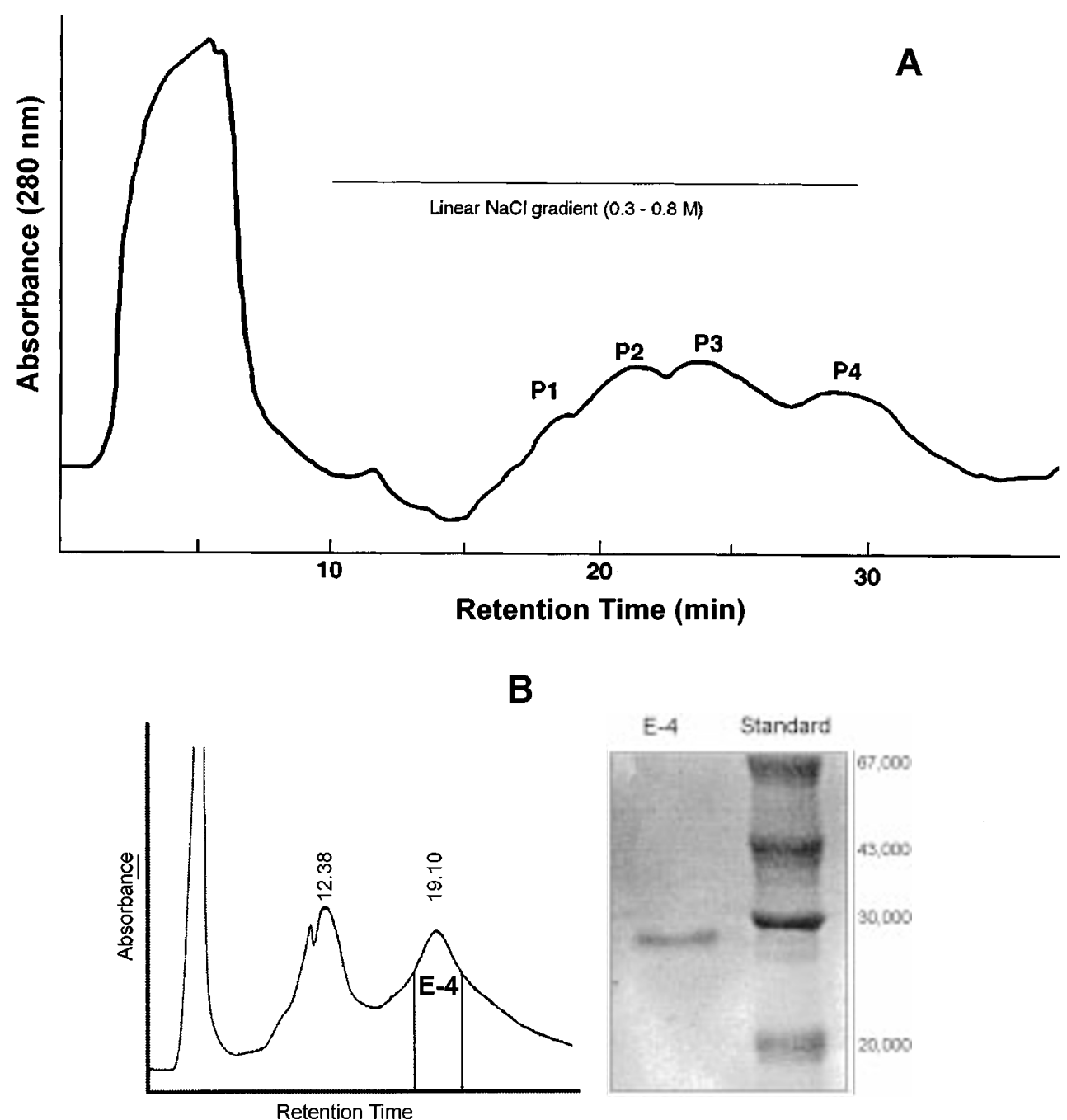

Figure 1. A, Purification of HNElastase by $\mathrm{CM}$-Sephadex ion exchange chromatography with shallow salt gradient $(0.3-0.8 \mathrm{M} \mathrm{NaCl})$ : Experimental details are in the Methods. B, For Raman experiments, HNElastase (P-4) was chromatographed again on HPLC with TSK-250 column. Mobile phase was 50 mM Tris$\mathrm{Cl}$ containing $0.8 \mathrm{M} \mathrm{NaCl}$ at pH 7.3. Inserted figure showed the single band of HNElastase that was purified by HPLC with gel filtration column chromatography. 

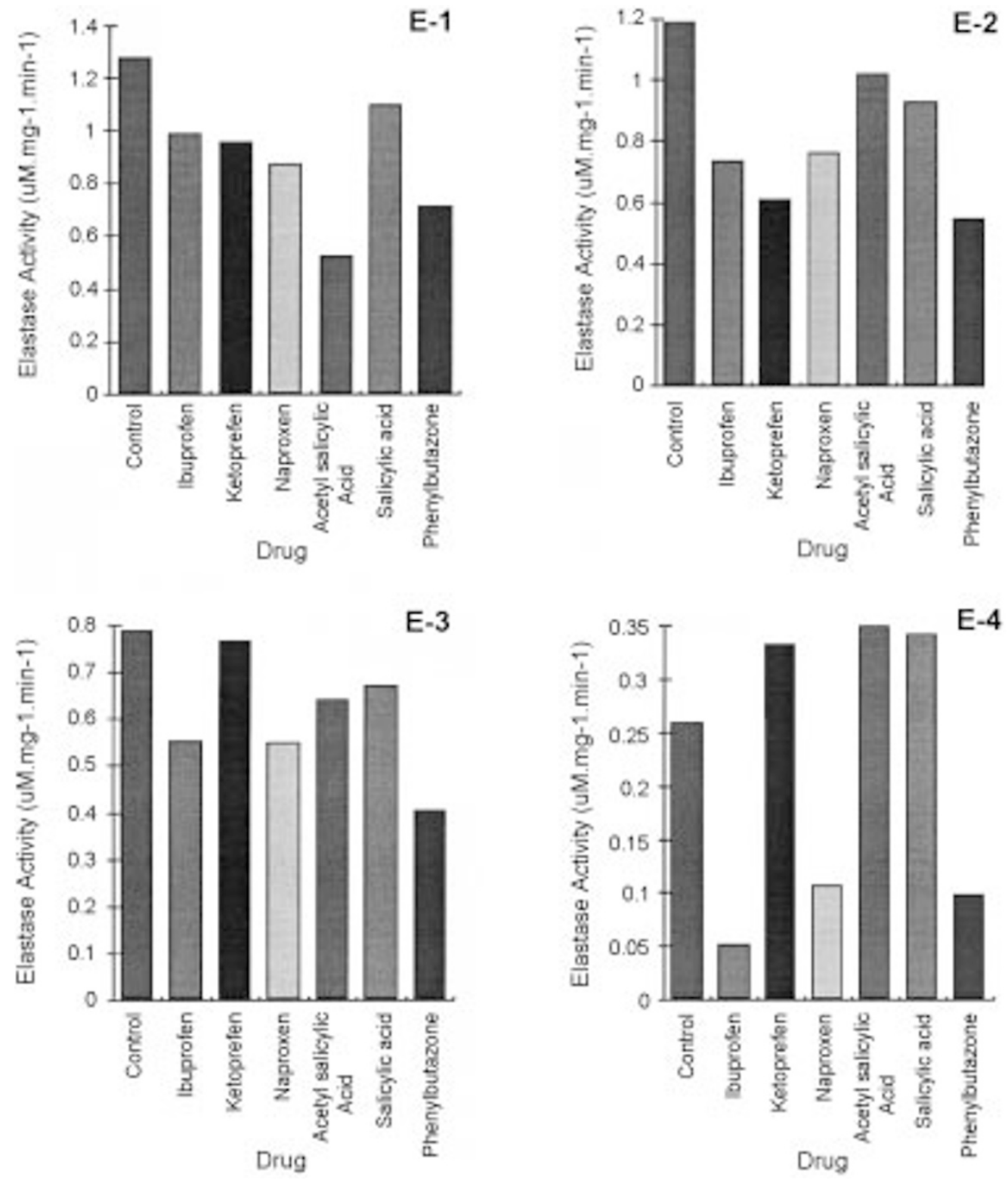

Figure 2. Inhibition of individual HNElastase isozymes by NSAIDs: Experimental details are in the Methods. E-1, E-2, E-3 and E-4 are the HNElastase isozymes that separated by CM-Sephadex ion exchange chromatography. Effects of NSAIDs on the activity of HNElastase were tested only the drugs that were soluble in $20 \%$ of DMSO.

inhibition characteristics by various NSAIDs (drug concentration: $0.5 \mathrm{mM}$ ); i.e., E-1, E-2, and E-3 were inhibited well by ibuprofen, ketoprofen, naproxen, phenylbutazone, salicylic acid, and acetyl salicylic acid. However, E-4 was inhibited by ibuprofen, naproxen, and phenylbutazone, but not by ketoprofen, salicylic acid, and acetyl salicylic acid. Phenylbutazone $(0.5 \mathrm{mM})$ inhibited all four HNElastase isozymes as much as about $50 \%$ of control activity, and the inhibition was concentration dependent manner up to $2 \mathrm{mM}$. Therefore, we employed phenylbutazone to investigate and explore the molecular pharmacological mechanism of the inhibition of
NSAIDs.

\section{Raman shift of HNElastase-phenylbutazone-complex}

Binding of phenylbutazone to the human neutrophil elastase molecules was stable, and the HNElastasephenylbutazone complex was not dissociated during the sample mixture that was dialyzed to remove unbound phenylbutazone from the complex.

Raman spectra of HNElastase with or without binding of phenylbutazone are shown in Figure 3. In Figure 3, A, $B, C$, and $D$ indicate the Raman spectra of control HNElastase, HNElastase-phenylbutazone complex, sus- 


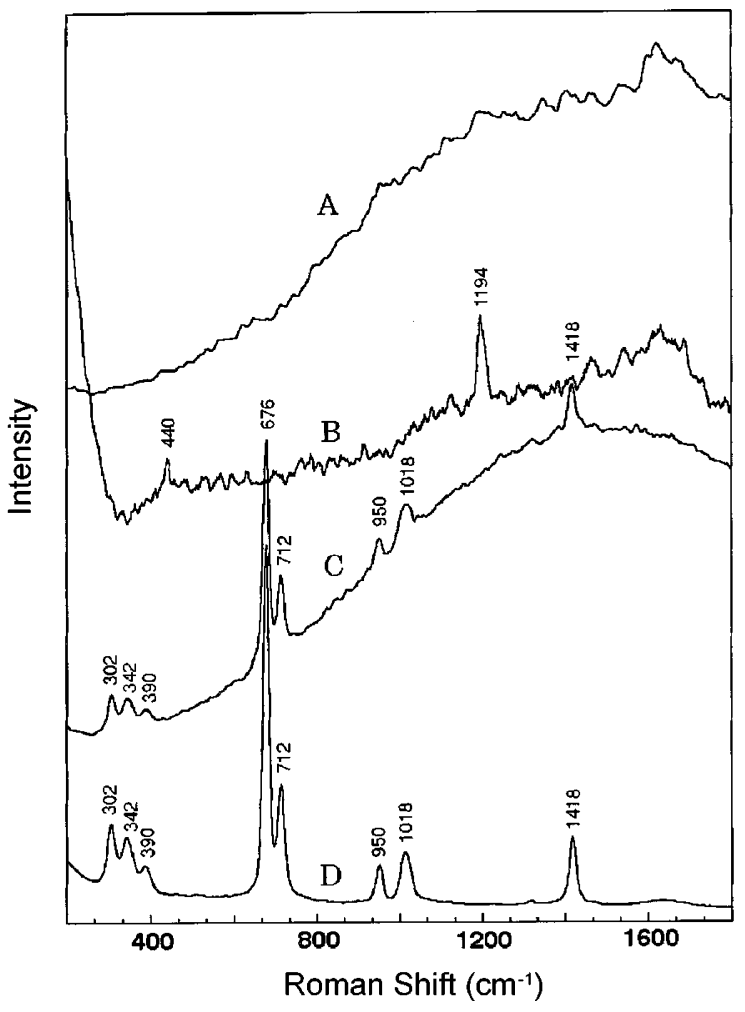

Figure 3. Raman spectra of elastase, elastase-phenylbutazone-complex, mixture of elastase phenylbutazone, and medium: Experimental details of preparation of the samples were described in the "Methods". Sample $\mathbf{A}$ and B were dialyzed against $50 \mathrm{mM}$ Tris- $\mathrm{Cl}, \mathrm{pH}$ 7.3. A, control elastase; B, HNElastase-phenylbutazone complex; $\mathbf{C}$, suspended elastase in the reaction medium (D) containing phenylbutazone; $\mathbf{D}$, reaction medium containing dimethylsulfoxide (while elastase reacted with phenylbutazone, $20 \%$ of dimethylsulfoxide was added in the reaction medium for prevention of precipitation).

pension of HNElastase and phenylbutazone respectively in the reaction medium, and medium (20\% DMSO) only. Raman spectra of reaction medium containing $20 \%$ of dimethylsulfoxide only showed several very intense bands $\left(676,712 \mathrm{~cm}^{-1}\right)$ and strong or medium intense bands such as $302,342,390,950,1018,1418 \mathrm{~cm}^{-1}$ (Figure 3D). Comparison of the Raman spectra of HNElastase (Figure 3A) with HNElastase-phenylbutazone complex (Figure 3B) showed similar contour but unique Raman shift bands between 200 and $1800 \mathrm{~cm}^{-1}$. The new bands at 200, 440, and $1194 \mathrm{~cm}^{-1}$ appeared in the HNElastase-phenylbutazone complex (Figure 3B), but not in the control. Figure $3 \mathrm{C}$ is the Raman spectrum of freshly prepared mixture of elastase and phenylbutazone (without incubation, simply suspended in the reaction medium containing phenylbutazone) without dialysis. The contour of $3 \mathrm{C}$ is similar to that of control elastase (3A) but Raman bands are similar to that of the medium (3D). However, this spectrum (3C) did not show the

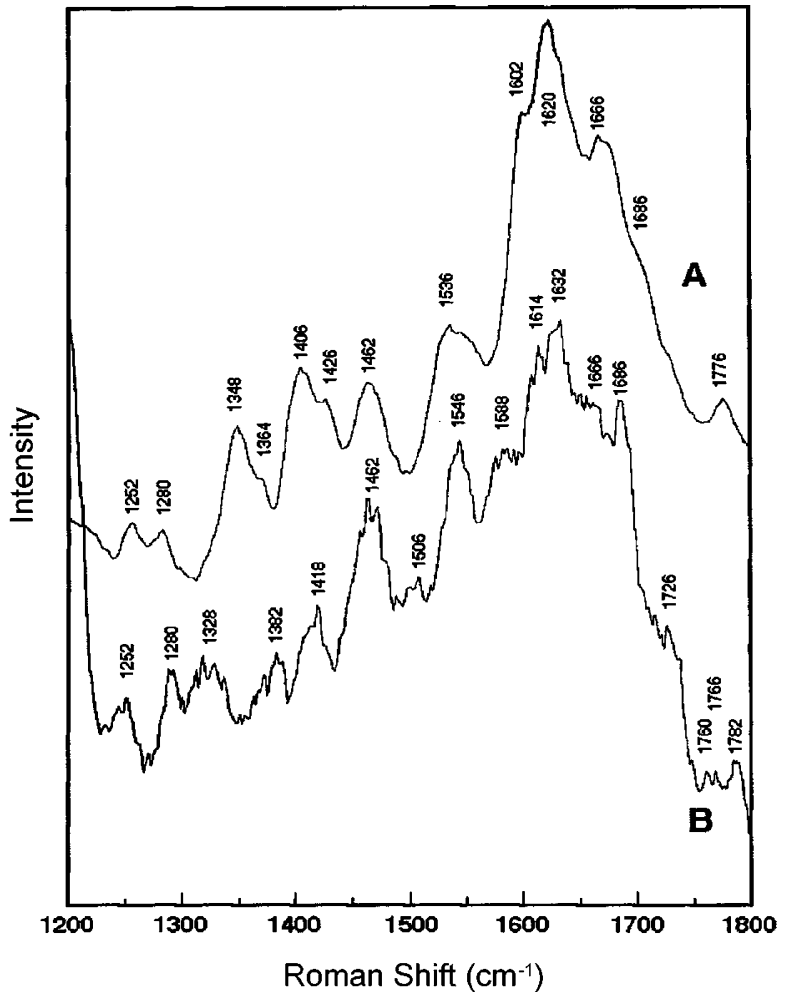

Figure 4. Raman spectra of control and elastase-phenylbutazone complex: Samples were scanned at wave number $1100-1800 \mathrm{~cm}^{-1}$. Experimental details of preparation of the samples were described in the "Methods". Sample A and B were dialyzed against $50 \mathrm{mM}$ Tris-Cl, pH 7.3. A, control elastase; B, human neutrophil HNElastase-phenylbutazone complex.

major strong Raman bands at 440 and $1194 \mathrm{~cm}^{-1}$ that showed in the HNElastase-phenylbutazone complex. Details of the Raman frequencies of control- and HNElastase-phenylbutazone complex are shown in Figure 4.

The comparison of two Raman spectrum (Figure 4) between wave number 1200 and $1600 \mathrm{~cm}^{-1}$. HNElastase-phenylbutazone complex showed new bands, which were not in the control HNElastase, at 1194, 1328, $1382,1418,1506$, and $1588 \mathrm{~cm}^{-1}$. Especially a band at $1194 \mathrm{~cm}^{-1}$ was very strong and unique in HNElastasephenylbutazone complex. In Raman spectrum between 1600 and $1800 \mathrm{~cm}^{-1}$, HNElastase-phenylbutazone complex showed several new bands such as wave numbers 1614, 1632, 1726, and $1782 \mathrm{~cm}^{-1}$, and band $1666 \mathrm{~cm}^{-1}$ became smaller than control and band $1686 \mathrm{~cm}^{-1}$ was larger than control. In the HNElastase-phenylbutazone complex, band $1348 \mathrm{~cm}^{-1}$ was disappeared. There were some Raman shifts after drug bound to the elastase such as band $1406 \mathrm{~cm}^{-1}$ shifted to $1418,1536 \mathrm{~cm}^{-1}$ to $1546 \mathrm{~cm}^{-1}, 1620$ to $1632 \mathrm{~cm}^{-1}$. The weak band 1686 $\mathrm{cm}^{-1}$ became stronger and band $1770 \mathrm{~cm}^{-1}$ became weaker, and new band $1782 \mathrm{~cm}^{-1}$ appeared in the HNElastase-phenylbutazone complex. 


\section{Discussion}

Three step procedure includes Ultrogel AcA 54 gel filtration, CM-Sephadex ion exchange chromatography (linear salt gradient $0.15-1 \mathrm{M} \mathrm{NaCl}$ ), and shallow salt gradient ion exchange chromatography (salt gradient $0.3-0.8 \mathrm{M} \mathrm{NaCl}$ ). It is an effective method and the last step is an important step for purification and it is a new method ever reported for purification of HNElastase isozymes. Addition of dithiothretol (DTT, final concentration: $2 \mathrm{mM}$ ) to the mobile phase prevented aggregation or dimerization of the isozymes, and increased efficiency of purification and purity of the enzyme. For the Raman experiments, the four elastase isozyme, E-4 was purified by an additional step of purification by gel filtration column on HPLC equilibrated with $0.8 \mathrm{M} \mathrm{NaCl}$. The enzyme was eluted in a single symmetrical peak (Figure 1B).

Four different HNElastase isozymes (E-1, E-2, E-3, E4) that were partially purified by CM-Sephadex ion exchange chromatography with shallow salt gradient (0.3$0.8 \mathrm{M}$ of $\mathrm{NaCl}$ ) showed different elastase inhibition characteristics. Figure 2 showed that four isozymes revealed different inhibition characteristics by various NSAIDs(at constant drug concentration: $0.5 \mathrm{mM}$ ); i.e., E-1, E-2, and E-3 were inhibited well by ibuprofen, ketoprofen, naproxen, phenylbutazone, salicylic acid, and acetyl salicylic acid. However, E-4 was inhibited by ibuprofen, naproxen and phenylbutazone but not by ketoprofen, salicylic acid and acetyl salicylic acid. Drugs such as ibuprofen, ketoprofen, naproxen, phenylbutazone, salicylic acid, and acetyl salicylic acid inhibited HNElastase in concentration dependent manner. Ibuprofen, ketoprofen, naproxen, phenylbutazone showed partial competitive inhibition (data not shown). The molecular structure of HNElastase constructed by molecular visualization system and crystallographic data allowed to view a unique active site (Kang, 1998). Molecular structural characteristics of HNElastase are; 1) the core area of elastase molecule was structurally stable, 2) the active site was at the core of the molecule, 3) the active center was in the deep pocket, 4) the rim of the pocket was surrounded by hydrophobic amino acids such as Phe170, Gly171, Phe25, Leu20, Val46, Tyr80, Pro82, and Leu85, 5) at the nearest neighboring area of active site, there were one positive charged atom (nitrogen atom of His41) and two negative charged atoms (sulfur atoms of Cys26 and Cys 42). The partial competitive inhibition by the drug molecules appeared to be derived from by an ionic interaction at an adjacent active site and partial covering up of the active site by hydrophobic interaction between the side chain of drug molecule and the hydrophobic amino acids surrounded the active site. The hydrophobicity may hinder free competition between inhibitor and substrate and it may cause a partial competitive inhibition. On the other hand, salicylic acid, and acetyl sali- cylic acid inhibited HNElastase competitively (data not shown). This competitive inhibition might come from pure classical competition between salicylate and substrate at the active site without hindrance of additional hydrophobic interaction. We, therefore, suggest that there must be two different classes of NSAIDs, i.e., elastase sensitive and insensitive drugs. Therefore, clinical application of the elastase sensitivity will be another important factor for the treatment of tissue destructive inflammatory disease and for the development of new drugs.

Phenylbutazone $(0.5 \mathrm{mM})$ inhibited all four HNElastase isozymes effectively, and the inhibition was concentration dependent up to $2 \mathrm{mM}$. Therefore, we started to investigate the molecular pharmacological mechanism of the inhibition with phenylbutazone. Tolmetin and oxyphenbutazone were precipitated in our reaction medium containing DMSO (final concentration: $25 \%$ ), preventing a further investigation on the inhibition characteristics under this medium condition.

Raman frequencies of HNElastase also undergo drastic changes upon binding of phenylbutazone. The differences between Figures $A$ and $B$ in Figures 3 and 4 must reflect the effects of the enzyme complex formation with phenylbutazone. In the elastase-phenylbutazone complex, the Raman shift at $1194 \mathrm{~cm}^{-1}$ could be the evidence of the two $-\mathrm{N}-\Phi$ radicals of phenylbutazone molecule (Scheule et al., 1977 and 1979). The bands at 1328 and $1418 \mathrm{~cm}^{-1}$ in the elastase-phenylbutazone complex may be related to the molecular structural conformational change by DMSO. Band $1382 \mathrm{~cm}^{-1}$ in the phenylbutazone-elastase complex (Figure 4B) may be related to the presence of pyrazole ring in the complex as seen in the crystal and aqueous samples of azocarboxypeptidase (Scheule et al., 1977, 1979, 1980). Bands 1506, and $1588 \mathrm{~cm}^{-1}$ are new bands and may be related to the conformational change of carboxyl radical at an adjacent active site such as Asp88. Disappearance of 1404 may be related to the covering up of histidine ring at an area of active site (Lord, and Yu, 1970a).

Bands 1602, 1620, and $1782 \mathrm{~cm}^{-1}$ in HNElastase may be shifted to 1614,1632 , and $1788 \mathrm{~cm}^{-1}$, respectively, by formation of drug-elastase complex. Bands 1686, $1726 \mathrm{~cm}^{-1}$ were not shifted but amplified by complex formation with phenylbutazone. Band $1776 \mathrm{~cm}^{-1}$ was shrunken by formation of drug-elastase complex. We speculated that Raman shifts between 1600 and 1800 $\mathrm{cm}^{-1}$ related to the conformational change especially by carboxyl radicals, but it needs detail study in the future.

It has been known that human neutrophil elastase reacts with substrate at the active site as in the "Chargerelay" hypothesis of the mechanism of action of serine protease (Blow et al., 1969). The corresponding amino acids are Ser173, Asp88, and His41, and positive charge will be relayed from hydrogen atom of Asp88 through His41 to Ser173. When we apply this classical 


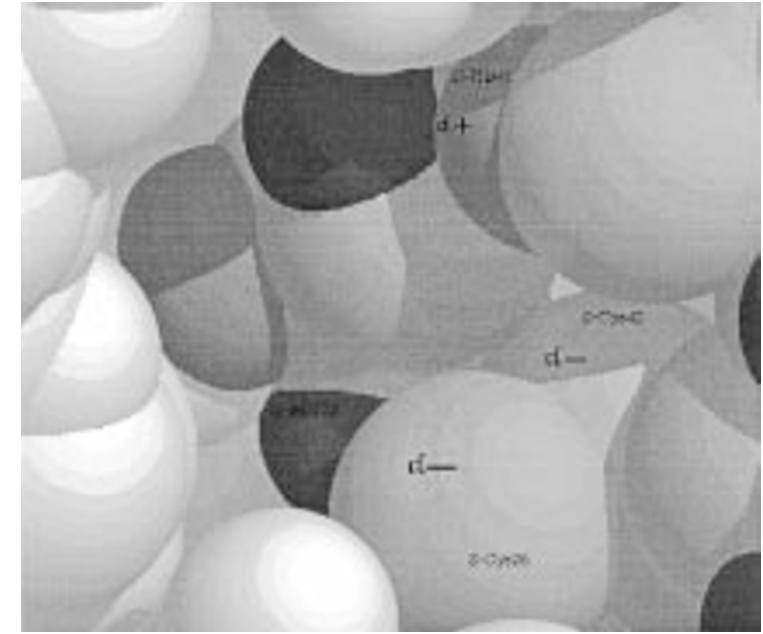

Figure 5. Magnified structure of active site: Ser173, His41, and Cys26 \& Cys42 formed -S-S- bond. Red, violet, gray, and yellow colored atoms are oxygen, nitrogen, carbon, and sulfur atoms, respectively. N-His-41 indicated the nitrogen atom in the ring histidine 41 molecule. O-Ser-173 indicated the oxygen atom in the serine 173 . S-cys-26 and S-cys-42 indicated the sulfur atoms in the cystine 26 and 42 , respectively.

mechanism of action to our experimental results, the inhibitory effects of human neutrophil elastase must be related to the property of the drug binding to the active site of the elastase such as complete or partial masking of the active site (Figure 5) (Kang, 1998). In phenylbutazone inhibition, both $-\mathrm{C}=\mathrm{O}^{\delta-}$ portions and nitrogen atoms of the pyrazole ring in the phenylbutazone molecule might be important factors for the binding of phenylbutazone molecule to the active site of the human neutrophil elastase molecule (Figure 6A).

The negative charged oxygen atoms in the $-\mathrm{C}=\mathrm{O}^{\delta-}$ portion of pyrazole ring may interact with the weak positive charged nitrogen atoms of the histidine ring of the active site (Figure 5). As one can see in the molecular models constructed by the crystallographic PDB data in previous paper (Kang, 1998), active sites of human neutrophil elastase showed important molecular structural factors; 1) the vicinity of the active site of human neutrophil elastase is surrounded by hydrophobic amino acids such as Phe, Gly, Leu, Val, Pro, and Leu, 2) one positive charged atom (nitrogen atom of His) and two negative charged atoms (sulfur atoms of Cys26 and Cys 42) at near the active site formed rigid conformation (Figure 6B). Therefore, three weak charged atoms might be bound electrostatically to the corresponding opposite charge of the phenylbutazone molecule, i.e., one of the two negative charged $\mathrm{C}=\mathrm{O}^{\delta-}$ and two positive charged $-\mathrm{N}+$ in the pyrazole ring of the phenylbutazone molecule (Figure 6B), leading to a strong interaction between the drug and enzyme.

Phenylbutazone may form key and lock like binding to the atoms charged with opposite charge near the active site of enzyme molecule. Furthermore, the surrounding

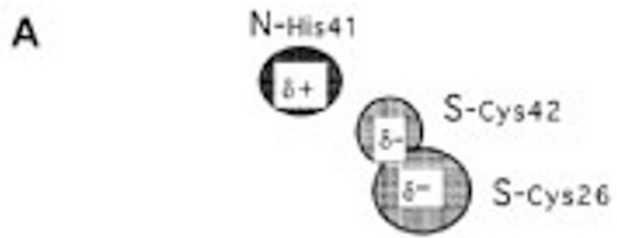

Charged atoms at near the active site

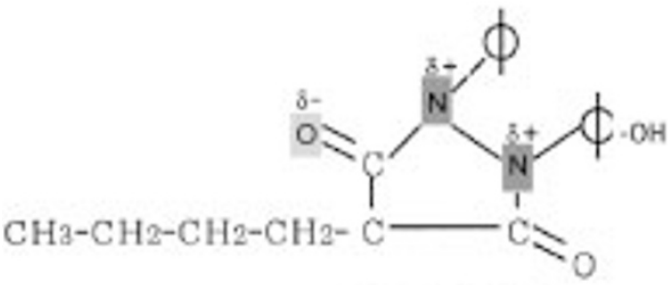

Phenyllbatazone

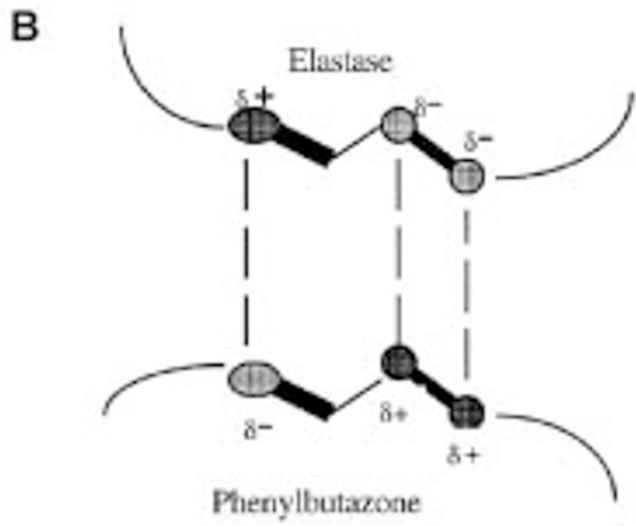

Figure 6. A, Schematic expression of charged atoms at the active site of elastase and phenylbutazone. B, Suggested model of the binding of phenylbutazone to the human neutrophil elastase: Details are in the text.

hydrophobic amino acid molecules of enzyme will also interact very effectively with the hydrophobic parts of the phenylbutazone such as benzene rings and aliphatic carbon chain. Binding of phenylbutazone to an area near the active site of human neutrophil elastase may cause masking of the active site with drug preventing the substrate from approaching the active site, possibly inhibiting the elastase activity. The interaction of the drug molecule to the active site by hydrophobicity will hinder the free competition between phenylbutazone and substrate, and as the result, phenylbutazone may inhibit HNElastase by partial competition. This result can also be applied to the development of new drug design.

In conclusion, the inhibitory effect of elastase could be, besides inhibition of cyclooxygenase, an another important mechanism of action of NSAIDs including aspirin, salicylate, and phenylbutazone in the treatment of inflammatory diseases. The mechanism of the elastase inhibition may be related to the specific molecular struc- 
ture of elastase and NSAIDs such as phenylbutazone, and the charged portion and hydrophobicity must be important factors for the drug binding to the active site of human neutrophil elastase.

\section{Acknowledgement}

This work was supported by grants from Basic Medical Science, Department of Education, Government of Republic of Korea, Medical Research Grant of Kosin University Medical College, and Specialized Center of Research (SCOR) Grant (2-P50-AR39239) National Institute of Health, U.S.A.

\section{References}

Abramson, S. R. and Weissman, G. (1989) The mechanism of action of nonsteroidal anti-inflammatory drugs. Arthritis Rheum. 32: 1-9

Baugh, R. J and Travis, J. (1976) Human leukocyte granule elastase: Rapid isolation and characterization. Biochemistry 15: $836-841$

Bernstein, F. C., Koetzle, T. F., Williams, G. J. B., Meyer, Jr. E. F., Brice, M. D., Rodgers, J. R., Kennard, O., Shimanouchi, T. and Tasimi, M. (1977) The Protein Data Bank: A computerbased archival file for macromolecular structures. J. Mol. Biol. 112: $535-542$

Blow, D. M., Birktoft, J. J. and Hartley, B. S. (1969) Role of a burried acid group in the mechanism of action of chymotrypsin. Nature 221: 337-430

Bode, W., Wei, A. Z., Buber, R., Meyer, E., Travis, J. and Newman, S. (1986) X-ray crystal structure of the complex of human leukocyte elastase (PMN elastase) and the third domain of the turkey ovomucoid inhibitor. EMBO J. 5: 14532458

Boyum, A. (1968) Separation of leukocytes from blood and bone marrow. Scan. J. Clin. Lab. Invest. Suppl. 97: 7

Cohen, A. B. (1975) The interaction of alpha-1-antitrypsin with chymotrypsin, trypsin and elastase. Biochim. Biophys. Acta 391: 193-200

Fritz, H., Jochum, M., Duswald, K. H., Dittmer, H., Kortmann, H., Neumann, S. and Lang, H. (1986) Granulocyte proteinases as mediators of unspecific proteolysis in inflammation; a review. In Cysteine Proteases and Their Inhibitor (Turk, V., ed) pp.785-807, Walter de Gruyter \& Co., Berlin

Insel, P. A. (1996) Analgesic-antipyretic and antiinflammatory agents and drugs employed in the treatment of gaut. In The Pharmacological Basis of Therapeutics (Hardman, J. G., Limbird, L. E., Molinoff, P. B., Rudden, R. W. and Gilman, A. G. eds) pp. 617-657. McGrew Hill, New York, NY

Janoff, A. (1972) Human neutrophil proteases in inflammation. Annu. Rev. Med. 23: 177-190

Jeung, H. Y., Ghim, S. Y. and Kang, K. (1987) Simultaneous separation of pure Neutrophils and lymphocytes from normal human blood by step gradient centrifugation. Korean $\mathrm{J}$. Immunol. 9: 27- 31

Kang, K. (1985) Characterization of human leukocyte elastase: Inhibition by chelators, role of zinc and calcium and effect of cations and anions on activity. University Microfilms International 84-25326

Kang, K. (1998) Molecular pharmacological interaction of phenylbutazone to human neutrophil elastase. Korean J. Physiol. Pharmacol. 2: 385-393

Kang, K., Kim, W. M., Hong, I. S. and Lee, M. S. (1996) Inhibition of human neutrophil elastase by NSAIDs and inhibitors, and molecular pharmacological mechanism of the inhibition. Korean J. Pharmacol. 32: 425-431

Keller, S., Schrader, B., Hoffmann, A., Schrader, W., Metz, K., Rehlaender, A., Panke, J. and Ruwe, M. (1994) Application of near-infrared-fourier transform Raman spectroscopy in medical research. J. Raman Spectrosc. 25: 663-671

Lentini, A., Ternai, B. and Ghosh, P. (1987) Synthetic inhibitors of human granulocyte elastase part 4 . Inhibition of human granulocyte elastase and cathepsin $\mathrm{G}$ by non-steroidal antiinflammatory drugs (NSAIDs). Biochem. Intl. 15: 1069-1078

Lord, R. C. and Yu, N. T. (1970a) Laser-excited Raman spectroscopy of biomolecules. II. Native ribonuclease and $\alpha$ chymotrypsin. J. Mol. Biol. 51: 103-105

McFarland, J. T., Watters, K. L. and Peterson, R. L. (1975) Resonance Raman investigation of an enzyme-inhibitor complex. Biochemistry 14: 624-630

Navia, M. A., McKeever, B. M., Springer, J. P., Lim, T. Y., Williams, H. R., Fluder, E. M., Dorn, C. P. and Hoogsteen, K. $H$. (1989) Structure of human neutrophil elastase in complex with a peptide chloromethylketon inhibitor at 1.84 angstroms resolution. Proc. Nat. Acad. Sci. USA 86: 7-11

Oholson, K. and Olsson, I. (1977) The extracellular release of granulocyte collagenases and elastase during phagocytic and inflammatory processes. Scand. J. Haematol. 19: 145-152

Salvesen, G. S. and Barrett, A. J. (1980) Covalent binding in their reaction with $\alpha 2-$ macroglobulin. Biochem. J. 187: 695701

Scheule, R. K., Van Wart, H. E., Vallee, B. L. and Scheraga, H. A. (1977) Resonance Raman spectroscopy of arsanilazocarboxypeptidase A: Determination of the nature of the azotyrosyl-248, zinc complex. Proc. Nat. Acad. Sci. USA 74: 3273-3277

Scheule, R. K., Van Wart, H. E., Zweifel, B. O., Vallee, B. L. and Scheraga, H. A. (1979) Resonance Raman spectroscopy of arsanilazocarboxypeptidase A: Assignment of vibrations of azotyrosyl-248. J. Inorg. Biochem. 11: 283-2835

Scheule, R. K., Van Wart, H. E., Vallee, B. L. and Scheraga, H. A. (1980) Resonance Raman spectroscopy of arsanilazocarboxypeptidase A: Conformational equilibria in solution and crystal phases. Biochemistry 19: 759-761

Sinha, S., Watorex, W., Karr, S., Giles, J., Bode, W. and Travis, J. (1987) Primary structure of human neutrophil 
elastase. Proc. Nat'l Acad. Sci. USA 84: 2228-2232

Starkey, P. M. (1980) The role of cellular elastases in inflammation. Front Matrix 8: 188-198

Vane, J. R. and Botting, R. M. (1987) Inflammation and the

mechanism of action of antiinflammatory drugs. FASEB J.1: 89-96Vane, J. R. and Botting, R. M. (1995) New insights into the mode of action of anti-inflammatory drugs. Inflamm. Res. 44: $1-10$ 